\title{
SARS-CoV-2 y sistema inmune: una batalla de titanes
}

Joel de León Delgado* 1; Arturo Pareja Cruz 1; Priscilia Aguilar Ramirez 1; Yanina Enriquez Valencia 1 ; Carlos Quiroz Carrillo ${ }^{1}$; Edward Valencia Ayala ${ }^{1}$

\section{RESUMEN}

La humanidad se enfrenta, en la actualidad, a un reto que no diferencia fronteras, ideologías, sistemas socioeconómicos, etnias, religiones o culturas. Es una guerra que nos une como especie biológica, en la que las armas fundamentales provienen de la investigación puesta al servicio de los sistemas de salud. En esta guerra los gobiernos y las organizaciones internacionales de salud definen la estrategia; mientras que los sistemas de salud y las fuerzas del orden, de la mano de la ciudadanía, concretan las acciones y libran cada una de las batallas. Sin embargo, el más importante de los enfrentamientos se realiza en el interior de cada uno de nosotros. Como Perseo enfrentó a Hades en una batalla de titanes para evitar que convirtiera la Tierra en un infierno, el sistema inmune pone en marcha una poderosa maquinaria en la que moléculas y células del sistema innato y adquirido actúan de manera coordinada para combatir al SARS-CoV-2. Una maquinaria que sigue un guion escrito por la evolución, y que deja en nuestro sistema de defensa una memoria que nos fortalecerá como especie para enfrentar futuros Hades. Este trabajo resume la valiosa información que se ha publicado en los últimos meses respecto al coronavirus SARS-CoV-2 en su interacción con el sistema inmune. Se incluyen aspectos relacionados con la detección de la respuesta inmune como herramienta para el diagnóstico de esta infección, y la manipulación del sistema inmune en la prevención o el tratamiento de la misma.

Palabras clave: Infecciones por coronavirus; Sistema inmunológico; Diagnóstico; Inmunoterapia (Fuente: DeSC BIREME).

\section{SARS-CoV-2 and the immune system: a battle of titans}

\author{
ABSTRACT
}

Humankind is currently facing a challenge that distinguishes no borders, ideologies, socioeconomic systems, ethnic groups, religions or cultures. It is a war that unites us as a biological species, in which the main weapons come from research aimed at improving health systems. In this war, governments and international health organizations define the strategy; and health systems and law enforcement agencies, together with citizens' support, carry out actions and fight the battles. However, the most important battle is fought inside each of us. Just like Perseus fought against Hades in a battle of titans to prevent the Earth from turning into hell, the immune system activates a powerful machinery in which molecules and cells of the innate and acquired immune system jointly act to defeat SARS-Cov-2: a machinery that follows a script written by evolution, and that will leave in our immune system a memory that will strengthen us as a species to face future Hades. This work summarizes valuable information published in recent months regarding the interaction between SARS-Cov-2 and the immune system. It also includes aspects related to the detection of the immune response as a tool for the diagnosis of this infection, as well as the manipulation of the immune system to prevent or treat the disease.

Keywords: Coronavirus infections; Immune system; Diagnosis; Immunotherapy (Source: MeSH NLM).

\footnotetext{
1 Universidad de San Martín de Porres, Facultad de Medicina Humana, Instituto de Investigación, Centro de Investigación de Infectología e Inmunología. Lima, Perú.

${ }^{*}$ Autor corresponsal
} 


\section{INTRODUCCIÓN}

En diciembre de 2019 se detectó en China el primer caso de infección con un nuevo coronavirus, posteriormente aislado, genéticamente caracterizado y denominado SARS-CoV-2 ${ }^{(1)}$. A pesar del impacto global de esta epidemia por su rápida expansión, no se trata de nuestro primer contacto, como especie, con esta familia de virus, que causa enfermedades zoonóticas ya que pueden transmitirse de algunos animales (aves y mamíferos, como los murciélagos) a los humanos ${ }^{(2)}$. Si consideramos esta característica, la amplia diversidad genética y la frecuente recombinación de estos genomas virales, no es raro que se detecten nuevos coronavirus en humanos, especialmente en comunidades donde existe un contacto cercano con los animales ${ }^{(3,4)}$.

Los coronavirus pueden producir cuadros clínicos que van desde el resfriado común hasta otros más graves como los que causan los virus del síndrome respiratorio agudo grave (SARS, por sus siglas en inglés) y el síndrome respiratorio de oriente próximo (MERS, por sus siglas en inglés) ${ }^{(5)}$. Estos otros coronavirus, aunque con una letalidad mayor que el SARS-CoV-2 ${ }^{(6)}$, tuvieron una expansión más limitada y, por ende, un impacto global menos significativo a nivel social y en los sistemas de salud. La vía principal de transmisión de los coronavirus en humanos es el contacto directo con gotas respiratorias a través de las mucosas de la boca, la nariz y los ojos ${ }^{(7)}$. No se descarta, sin embargo, la transmisión oral-fecal y la vertical de madre a feto, pero las evidencias hasta el momento no son suficientemente sólidas desde el punto de vista epidemiológico ${ }^{(8,9)}$.

Como el resto de los miembros de la familia de coronavirus, el SARS-CoV-2 tiene un genoma formado por una única cadena de ácido ribonucleico (ARN) de unos 30000 pares de bases, con características muy parecidas al ARN mensajero del hospedero. Esto último le permite adherirse directamente a los ribosomas e iniciar la traducción de sus proteínas estructurales y de aquellas requeridas para la replicación. Las glicoproteínas espiga de transmembrana (S) del SARS-CoV-2 han sido cristalizadas y su estructura y función dilucidadas; además, se ha confirmado su interacción con la enzima convertidora de angiotensina 2 humana (ACE2, por sus siglas en inglés) como vía de acceso a las células (10). Este hecho ha llevado a los investigadores a proponer estrategias de tratamiento basadas en la inhibición de este proceso molecular con el apoyo de agencias nacionales responsables del control de uso de medicamentos.

Sin embargo, un tema de especial relevancia en el control de este virus es la actividad del sistema inmune del hospedero. Los conocimientos básicos sobre la respuesta inmune antiviral describen al menos cuatro estrategias fundamentales para el control de la diseminación viral que involucran componentes celulares y moleculares del sistema innato y adquirido: 1) producción de interferones tipo I (IFN alfa y beta) que interfieren en los mecanismos de replicación viral; 2) producción de anticuerpos con capacidad de neutralizar la interacción de los virus con sus receptores celulares para limitar su acceso a las células hospederas; 3 ) activación de linfocitos asesinos naturales (NK, por sus siglas en inglés) con efecto citotóxico directo sobre células infectadas mediado por perforinas y granzimas que inducen apoptosis celular, y 4) activación de linfocitos T CD8+ también con actividad citotóxica específica sobre las células infectadas. Para lograr una respuesta de anticuerpos madura y de alta afinidad, así como para asegurar la efectiva activación de los linfocitos T CD8+, es determinante la cooperación de los linfocitos T CD4+ auxiliadores secretores de IFN gamma (Th1, del inglés $T$ helper 1).

A partir de estos conocimientos básicos, existen cinco elementos relevantes en la relación entre el SARS-CoV-2 y la respuesta inmune: 1) valorar cómo las herramientas moleculares y celulares del sistema inmune y su cinética de activación contribuyen al control de la infección con el SARS-CoV-2; 2) reconocer las estrategias de evasión del virus a la respuesta inmune, especialmente en las poblaciones vulnerables como los adultos mayores inmunosenescentes; 3) identificar inmunomarcadores con valor pronóstico; 4) valorar métodos de diagnóstico para tamizajes masivos basados en la determinación de la respuesta de anticuerpos, y 5) proponer estrategias de manipulación de la respuesta inmune para prevenir o tratar la infección. Estos aspectos guiaran la estructura de la esta revisión temática.

\section{RESPUESTA INMUNE CONTRA EL SARS-CoV-2}

\section{Respuesta inmune innata}

La activación de la respuesta inmune innata antiviral involucra la detección de los llamados patrones moleculares asociados a patógenos (PAMPs, por sus siglas en inglés). En este evento, los receptores tipo Toll (TLR, por sus siglas en inglés), presentes en diversas células del sistema inmune innato, son especialmente relevantes. Estas moléculas son un grupo de receptores, con alta homología estructural, que se expresan en la membrana plasmática o en el espacio intracelular, en dependencia del tipo de PAMPs que reconozcan. Específicamente, los TLR relevantes en la detección de ácidos nucleicos de origen viral son el TLR3 y el TLR7 ${ }^{(6,11)}$. La activación de estos receptores conduce a la estimulación de la respuesta inmune inflamatoria, mediada por citocinas como IL-1, IL-6 y el factor de necrosis tumoral alfa (TNFa, de sus siglas en inglés). Otras señales intracelulares activadas por estos receptores conducen la transcripción, traducción y secreción de IFNs tipo I ${ }^{(6,12)}$. Estas moléculas son muy relevantes en la inhibición de la replicación viral, y además contribuyen a la activación de la 
fagocitosis mediada por macrófagos y a la activación de la actividad citotóxica de los linfocitos NK. Otros receptores del sistema innato también contribuyen a activar la respuesta inmune antiviral, como los receptores tipo-NOD, tipo-RIG y tipo-lectinas C ${ }^{(11)}$.

Entre las células del sistema inmune innato relevantes en la respuesta antiviral merecen especial atención las células dendríticas. Este grupo, fenotípicamente diverso, se encarga de detectar la presencia de señales de peligro en los tejidos periféricos asociadas, en este caso, a los PAMPs virales, y trasladar esta información molecular a los órganos linfoides secundarios para estimular la respuesta inmune adaptativa. Para ello, ocurre un proceso de maduración celular que implica modificaciones morfofuncionales en estas células, de manera que ganan en capacidad migratoria, capacidad de presentación de antígenos en el complejo principal de histocompatibilidad (HLA I y II), expresión de moléculas de coestimulación y secreción de citocinas ${ }^{(11)}$. Estas células tienen dos habilidades muy especiales que las distinguen del resto de las células presentadoras de antígenos: 1) la capacidad única de activar la respuesta de linfocitos T vírgenes auxiliadores CD4+ y citotóxicos CD8+, y 2) la capacidad de realizar la presentación cruzada de antígenos, o sea, de endocitar antígenos extracelulares (como las células infectadas con virus o partículas virales), procesar estos antígenos y presentarlos tanto en HLA I como HLA II. Este último proceso ha sido fijado por la evolución como estrategia para activar la respuesta citotóxica de linfocitos T CD8+ contra células infectadas con el virus y también células neoplásicas. De hecho, los IFNs tipo I promueven la presentación cruzada de antígenos mediada por células dendríticas ${ }^{(13)}$.

\section{Respuesta inmune adquirida}

Los actores fundamentales en la respuesta antiviral por parte del sistema adquirido son los anticuerpos y los linfocitos $T$
CD8+. Aunque, como se mencionó anteriormente, la actividad auxiliadora de los linfocitos T CD4+ es muy relevante. Estos últimos determinan la activación del efecto citotóxico en los linfocitos T CD8+, la maduración de la respuesta de anticuerpos en los linfocitos B (incremento de la afinidad por hipermutación somática, cambio de clase de lgM a lgG), y adicionalmente, determinan el establecimiento de la memoria inmunológica, tanto de células T citotóxicas como de células secretoras de anticuerpos. Estudios previos respecto a los cuadros de SARS en modelos animales demostraron que, si bien el infiltrado inflamatorio en el intersticio pulmonar es fundamentalmente de linfocitos citotóxicos $T$ CD8+, la eliminación de los linfocitos T CD4+ produce una severa neumonitis como consecuencia de la reducción de la producción de anticuerpos anti-SARS efectivos y de la migración de linfocitos citotóxicos a los pulmones, lo que retrasa el aclaramiento de la carga viral ${ }^{(14)}$.

La activación de linfocitos $B$ y la consecuente respuesta de anticuerpos son muy relevantes en el control de la infección viral. Respecto a los coronavirus, se ha demostrado que las glicoproteínas $S$ son altamente inmunogénicas, y se ha conseguido mapear para el agente causante del MERS, un epítopo lineal para el virus con alta capacidad de activar la respuesta de linfoctos $B$ y $T^{(15)}$. La seroconversión tardía de pacientes con cuadros de SARS y MERS se relacionó con mal pronóstico, lo que evidenciaba la relevancia de los anticuerpos en la respuesta antiviral contra estos virus. Generalmente, esta se logra en un plazo de entre 4 días y 14 días con anticuerpos lgG neutralizantes en circulación por hasta dos años ${ }^{(16)}$. Respecto al SARS-CoV-2 quedan estudios por realizar para determinar el valor predictivo de la respuesta humoral mediada por anticuerpos, tanto de la seroconversión como de su cinética. Sin embargo, la capacidad del virus de estimular la seroconversión y la producción de anticuerpos neutralizantes está confirmada (6) Las características generales de la respuesta inmune innata y adquirida antiviral se describen en la figura 1.

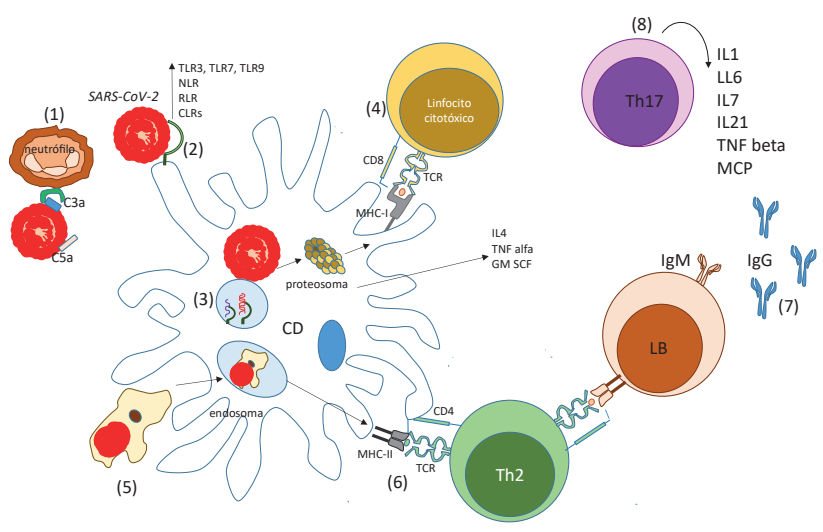

Figura 1. Respuesta inmune innata y adquirida en la infección por SARS-CoV-2: 1) los neutrófilos reconocen al SARS-CoV-2 por medio de los receptores CR1 y CR2, activados por las anafilotoxinas C3a y C5a del sistema complemento humano; 2) las células dendríticas reconocen al virus a través de los receptores de reconocimiento de PAMPs (TLR, NLR, RLR y CLPs); 3 ) en los endosomas se genera una respuesta parcial 
del sistema inmune al activarse TLR3 y TLR7 con el ARN viral, lo que desencadena las vías de señalización que inducen la producción de IFNs tipo I y citocinas proinflamatorias; 4) la fagocitosis de células infectadas con el virus y la presentación cruzada de antígenos virales en HLA I estimula a los linfocitos citotóxicos T CD8+; 5) la presentación de antígenos virales en HLA ll estimula a los linfocitos auxiliadores T CD4+; 6 y 7) estos linfocitos cooperan con los T CD8+ e instruyen a los linfocitos B para producir anticuerpos de alta afinidad contra epítopos del SARS-CoV-2, y 8) además, se activan y diferencian linfocitos T proinflamatorios tipo Th17.

\section{SARS-COV-2, INMUNOPATOGENIA E INMUNOEVASIÓN}

Al igual que otras familias virales, los coronavirus han desarrollado estrategias de inmunoevasión a partir de la presión selectiva de los mecanismos efectores del sistema inmune. Estas estrategias influyen en la eficacia de las respuestas innata y adaptativa, lo puede justificar, parcialmente, tanto el largo periodo de incubación relativo a la influenza ${ }^{(17)}$ como la mayor susceptibilidad de individuos con condiciones crónicas preexistentes y adultos mayores (18). En general, el incremento del índice neutrófilos/linfocitos con base en una linfopenia y la ocurrencia de un síndrome de liberación de citocinas proinflamatorias son eventos relacionados con la severidad y mortalidad de la enfermedad (18) y también han sido determinados en las epidemias de SARS y MERS $(19,20)$. Este proceso inflamatorio se ha relacionado con diversos mecanismos, incluyendo tanto la modulación negativa de la expresión de ACE2 en el epitelio pulmonar como el incremento de la secreción de esta enzima, eventos ambos considerados estimuladores de la respuesta inflamatoria (21).

Respecto a los IFNs tipo I, tanto en el SARS como el MERS, los virus, suprimen la secreción y el efecto de estas citocinas a través de diferentes mecanismos (22), lo que ha sido relacionado con la severidad de la enfermedad (23) (Figura 2). En este caso, el incremento de la letalidad por neumonía aumenta significativamente en un modelo murino con producción desregulada de IFNs tipo I y neutrofilia en pulmón ${ }^{23)}$. La infección con coronavirus reduce la expresión de HLA en las células dendríticas y los macrófagos $y$, por tanto, la presentación de antígenos a los linfocitos $\mathrm{T}^{(24)}$.

Un elemento muy interesante relacionado con la inmunopatogenia de los coronavirus, demostrado en diferentes modelos animales y en pacientes con SARS, es el efecto nocivo de los anticuerpos antiglicoproteína S. En este sentido, se han descrito dos situaciones diferentes: 1 ) la presencia de anticuerpos con capacidad de desencadenar efecto inflamatorio e injuria pulmonar, relacionados con la unión a receptores Fc en los macrófagos alveolares. La detección temprana en el suero de anticuerpos IgG neutralizantes se ha relacionado con mayor mortalidad en pacientes con SARS ${ }^{(25)}$, y 2) la amplificación dependiente de anticuerpo ( $A D E$, por sus siglas en inglés). El ADE ocurre en diferentes infecciones virales, como la del dengue, y se produce por la producción de niveles subóptimos de anticuerpos neutralizantes, lo que favorece la persistencia de la replicación viral y la inflamación (21).

Aunque varios de los aspectos mencionados son el resultado de investigaciones en modelos animales o de la experiencia del manejo de pacientes con SARS y MERS es importante tenerlos en consideración dentro de las alternativas terapéuticas como el uso de inmunoglobulina intravenosa para bloquear los receptores $\mathrm{Fc} y / 0$ el uso sistémico de drogas antinflamatorias y corticosteroides ${ }^{(21)}$.

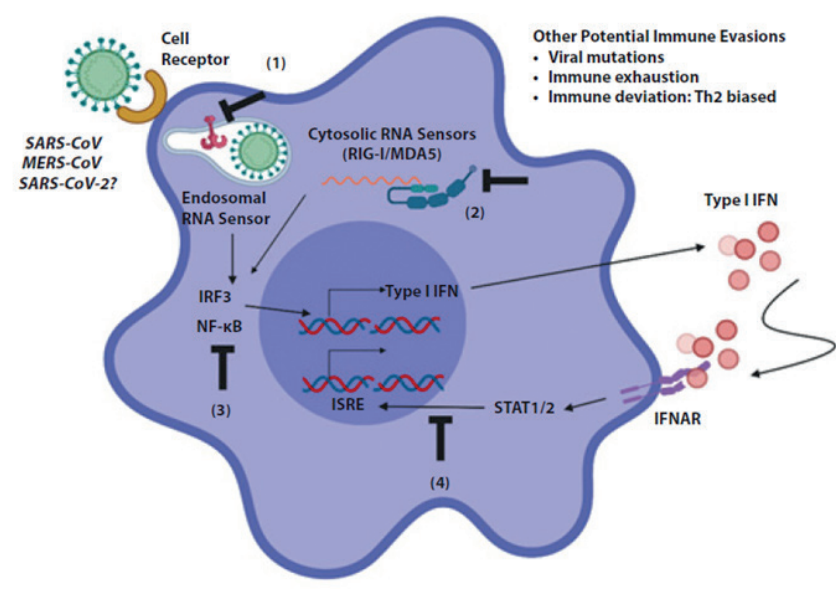

Figura 2. Mecanismos potenciales de inhibición de la producción y actividad de los IFNs tipo I por coronavirus. (1 y 2) Inhibición de la detección del ARN por los TLRs y otros receptores de PAMPs. (3) Bloqueo de las señales que estimulan la transcripción de los IFNs tipo I. (4) Inhibición de las vías de señalización de los IFNs tipo I. Modificado de Prompetchara E, 2020. 


\section{SENESCENCIA INMUNOLÓGICA Y SARS-COV-2}

El concepto de senescencia inmunológica trasciende las características del sistema inmune de los adultos mayores, para mostrar un fenómeno asociado a la composición y función del sistema inmune en diversas condiciones patológicas. Este concepto se ha operacionalizado con la determinación de marcadores celulares de agotamiento celular, marcadores de inflamación sistémica y alteraciones de la respuesta inmune.

En el caso del SARS-CoV-2 convergen ambas condiciones: por una parte, los adultos mayores son más susceptibles a la infección con tasas de mortalidad que multiplican varias veces la de los adultos jóvenes (26); por otra parte, se ha demostrado el agotamiento de poblaciones tan importantes para el control viral como las NK y los linfocitos T CD8+ mientras que se produce una marcado incremento en la frecuencia de neutrófilos, en correspondencia con lo que se indicó anteriormente (27). Se demostró una relación significativa entre la severidad de la enfermedad y la reducción de la frecuencia de linfocitos NK y T CD8+, aunque en general la reducción se produce en todos los pacientes infectados respecto a los controles sanos. Esta linfopenia está acompañada, además, del incremento en la expresión del receptor inhibitorio NKG2A, lo que produce una alteración de la función celular al limitar la producción de citocinas y granzimas ${ }^{(27)}$. La recuperación de los pacientes restaura los niveles celulares y reduce la expresión de NKG2D. Este resultado abre las puertas al potencial uso de bloqueadores de puntos negativos del control de la inmunidad como estrategia para potenciar la respuesta antiviral frente al SARS-CoV-2.

\section{MARCADORES INMUNOLÓGICOS EN EL DIAGNÓSTICO Y PRONÓSTICO DE SARS-COV-2}

La identificación de marcadores inmunológicos con valor diagnóstico, y especialmente pronóstico, en la infección con SARS-CoV-2 es muy relevante. Hasta el momento hay dos estrategias fundamentales relacionadas con el diagnóstico de esta infección: 1) una prueba rápida que determina la presencia de anticuerpos anti-SARS-CoV-2 y 2 ) una prueba molecular basada en la detección genética del virus a través de la reacción en cadena de la polimerasa cuantitativa en tiempo real (RT-PCR, por sus siglas en inglés). El reto de estas pruebas está en diagnosticar pacientes asintomáticos, pero infectados y con potencial infectivo. Aunque el RT-PCR es una técnica de alta sensibilidad y especificidad, se ha reportado que entre el 11 y $16 \%$ de los pacientes con resultados negativos por ensayo de RTPCR resultan posteriormente positivos a la infección. Además, más del $90 \%$ de los pacientes inicialmente negativos por RT-PCR tenían evidencias imagenológicas de la enfermedad ${ }^{(28)}$. Lo anterior ha permitido proponer una ventana de especificidad/sensibilidad para esta prueba.
En este sentido, la calidad de la toma y manipulación de la muestra son elementos importantes a considerar ${ }^{(28)}$. Se maneja el criterio de que el alta del paciente se realice después de dos muestras negativas al RT-PCR.

En lo referente al diagnóstico rápido, con base en presencia de anticuerpos anti-SARS-CoV-2, las características de la prueba (costo, manipulación, tipo de muestra, rapidez del resultado) ofrecen la posibilidad de un tamizaje en comunidades con alto riesgo de infección y en estudios epidemiológicos para detectar pacientes con poca sintomatología, pero con capacidad infectiva. Como se mencionó anteriormente, la seroconversión se produce en la mayoría de los pacientes, aunque, como es de esperar, en la respuesta inmune hay un retardo entre la producción de anticuerpos IgM e IgG neutralizantes. Por ello, el uso de pruebas que detecten ambos tipos de anticuerpos es particularmente valioso. Sin embargo, debido a la homología de secuencia entre las proteínas más inmunogénicas de los agentes causales de SARS y MERS, SARS-CoV-2 y otros coronavirus, debe considerarse la presencia de anticuerpos con reactividad cruzada. En general, ninguna de las pruebas empleadas en la actualidad aporta resultados totalmente concluyentes acerca de la presencia o ausencia de esta infección, por tanto, se recomienda su repetición en pacientes sospechosos.

Respecto a los marcadores inmunológicos pronósticos, se pueden considerar relevantes el título y capacidad neutralizante de los anticuerpos, la cinética de seroconversión, los niveles de IFN tipo I circulantes, la ocurrencia de linfopenia y el incremento en el índice neutrófilos/linfocitos. Estos aspectos deben tener en cuenta las diferencias demostradas entre pacientes y enfermos, y especialmente entre infectados con un curso grave o leve de la enfermedad. Además, la sensibilidad y simplicidad de las determinaciones es otro criterio a considerar para la realización de estas mediciones.

\section{ESTRATEGIAS DE INMUNOTERAPIA PARA SARS-COV-2}

Desde la detección del SARS-CoV-2 se han evaluado diversas alternativas vacunales para controlar de manera segura y eficaz la infección con este virus. Experiencias previas con vacunas ensayadas en modelos animales para los virus causantes de SARS y MERS ha demostrado que la proteína espicular $S$ es inmunogénica e induce anticuerpos que previenen la infección ${ }^{(6,29)}$. La mayor parte de estas propuestas vacunales se ensayaron solamente en ensayos clínicos fase I ${ }^{(6,30,31)}$, y no se pudo confirmar su eficacia. Aunque se cuenta con esta experiencia previa, se ha considerado que el desarrollo de ensayos clínicos frente al virus SARS-CoV-2 empieza prácticamente desde cero.

Actualmente, más de 30 compañías farmacéuticas, además de la academia, están desarrollando plataformas vacunales 
basadas en ácidos nucleicos, vectores adenovirales y proteínas recombinantes. De acuerdo a la experimentación con los virus que causan SARS y MERS, la mayoría de las vacunas que se están proponiendo frente al SARS-CoV-2 están fundamentadas en la proteína $S$ que, como ya se mencionó, se encarga de mediar la fusión del virus a las membranas celulares a través de ACE2. En este sentido se ha considerado: 1) uso de proteína $S$ recombinante purificada, completa o un fragmento inmunogénico; 2) uso de proteína $\mathrm{S}$ expresada in vivo a partir de un virus recombinante, y 3 ) vacuna basada en ácidos nucleicos que expresen la proteína. Estas últimas son fáciles de fabricar y, en consecuencia, su desarrollo se encuentra avanzado. La empresa Moderna Therapeutics ensaya un candidato vacunal contra el COVID-19 basado en ARN mensajero sintético y se encuentra reclutando voluntarios sanos en EE. UU. (45 en total, entre 18 y 55 años) ${ }^{(32)}$.

Otra alternativa a considerar es la generación de anticuerpos monoclonales (AcMs) con capacidad de neutralización. El uso de bibliotecas con miles de millones de secuencias de la región de los anticuerpos humanos encargados de reconocer los antígenos se ha expresado en fagos. Este tipo de bibliotecas permite hacer la selección de los anticuerpos humanos que reconocen con alta afinidad a las proteínas virales e inhiben su capacidad infectiva. Esta estrategia se ensayó con el virus que causa el MERS y se obtuvo un AcM que reduce en hasta $90 \times 10^{3}$ veces el título de ARN viral a nivel de pulmones ${ }^{(33)}$.

Un tema no menos relevante es el desarrollo de un modelo animal que reproduzca la enfermedad humana que produce el SARS-CoV-2, lo que permitiría evaluar la eficacia y la toxicidad inducidas por terapias experimentales. Al considerar la relevancia de la proteína ACE2 para la infección del SARS-CoV-2, los animales transgénicos que expresan este receptor humano serían de gran utilidad ${ }^{(34)}$. Adicionalmente, se ha determinado la capacidad neutralizante de anticuerpos anti-proteína $\mathrm{S}$ utilizando modelos celulares in vitro ${ }^{(10)}$. La opción de utilizar el suero de pacientes infectados y recuperados de la enfermedad es una alternativa de inmunoterapia pasiva en evaluación.

\section{CONCLUSIONES}

La respuesta inmune efectiva contra el SARS-CoV-2 es determinante para su control a escala individual. Sin embargo, comprender los mecanismos de respuesta inmunológica contra este virus, su cinética y las estrategias virales para escapar del sistema inmune resulta determinante para la implementación de alternativas efectivas de diagnóstico, pronóstico, prevención y terapia, lo cual contribuirá al control de esta infección a escala poblacional.
Contribuciones de los autores: Joel de León, Arturo Pareja: Redacción, revisión bibliográfica, edición final y coordinación general. Priscilia Aguilar, Yanina Enrique, Carlos Quiróz, y Edward Valencia: Revisión bibliográfica y edición.

Fuentes de financiamiento: Este artículo ha sido financiado por los autores.

Conflicto de interés: Los autores declaran no tener ningún conflicto de interés.

\section{REFERENCIAS BIBLIOGRÁFICAS}

1. GenBank. Severe acute respiratory syndrome coronavirus 2 isolate Wuhan-Hu-1, complete genome. Disponible en: https://www. ncbi.nlm.nih.gov/nuccore/MN908947.3

2. World Health Organization. Novel Coronavirus (2019-nCoV) situation reports. Disponible en: https://www.who.int/ emergencies/diseases/novel-coronavirus-2019/situation-reports

3. Cyranoski D. Mystery deepens over animal source of coronavirus. Nature. 2020; 579(7797): 18-9.

4. Cui J, Li F, Shi Z-L. Origin and evolution of pathogenic coronaviruses. Nat Rev Microbiol. 2019; 17(3): 181-92.

5. Paules $\mathrm{Cl}$, Marston HD, Fauci AS. Coronavirus infections-more than just the common cold. JAMA. 2020; 323(8): 707-8.

6. Prompetchara E, Ketloy C, Palaga T. Immune responses in COVID-19 and potential vaccines: lessons learned from SARS and MERS epidemic. Asian Pac J Allergy Immunol. 2020; 38(1): 1-9.

7. Kai-Wang K, Tsang O, Chik-Yan C, Chan KH, Wu TC, Chan JM, et al. Consistent detection of 2019 novel coronavirus in saliva. Clin Infect Dis. 2020.

8. Yeo C, Kaushal S, Yeo D. Enteric involvement of coronaviruses: is faecal-oral transmission of SARS-CoV-2 possible?. Lancet Gastroenterol Hepatol. 2020; 5(4): 335-7.

9. Chen H, Guo J, Wang C, Luo F, Yu X, Zhang W, et al. Clinical characteristics and intrauterine vertical transmission potential of COVID-19 infection in nine pregnant women: a retrospective review of medical records. Lancet. 2020; 395(10226): 809-15.

10. Walls AC, Park Y, Tortorici MA, Wall A, MCGuire AT, Veesler D. Structure, function, and antigenicity of the SARS-CoV-2 spike glycoprotein. Cell. 2020.

11. Li G, Fan Y, Lai Y, Han T, Li Z, Zhou P, et.al. Coronavirus infections and immune responses. J Med Virol. 2020; 92(4): 424-32.

12. Ivashkiv LB, Donlin LT. Regulation of type I interferon responses. Nat Rev Immunol. 2014; 14(1): 36-49.

13. Le Bon A, Etchart N, Rossmann C, Ashton M, Hou S, Gewert D, et al. Cross-priming of CD8+ T cells stimulated by virus-induced type I interferon. Nat Immunol. 2003; 4(10): 1009-15.

14. Chen J, Lau YF, Lamirande EW, Paddock CD, Bartlett JH, Zaki $\mathrm{SR}$, et al. Cellular immune responses to severe acute respiratory syndrome coronavirus (SARS-CoV) infection in senescent BALB/C mice: CD4+ T cells are important in control of SARS-CoV infection. J Med Virol. 2010; 84(3): 1289-1301.

15. Ababneh M, Alrwashdeh M, Khalifeh M. Recombinant adenoviral vaccine encoding the spike 1 subunit of the Middle East Respiratory Syndrome Coronavirus elicits strong humoral and cellular immune responses in mice. Vet World. 2019; 12(10): 1554-62.

16. Liu W, Fontanet A, Zhang PH, Zhan L, Xin ZT, Baril L, et al. Twoyear prospective study of the humoral immune response of 
patients with severe acute respiratory syndrome. J Infect Dis. 2006; 193(6): 792-5.

17. Lessler J, Reich NG, Brookmeyer R, Perl TM, Nelson KE, Cummings DA. Incubation periods of acute respiratory viral infections: a systematic review. Lancet Infect Dis. 2009; 9(5): 291-300.

18. Huang C, Wang Y, Li X, Ren L, Zhao J, Hu Y, et al. Clinical features of patients infected with 2019 novel coronavirus in Wuhan, China. Lancet. 2020; 395: 497-506.

19. Mahallawi WH, Khabour OF, Zhang Q, Makhdoum HM, Suliman BA. MERS-CoV infection in humans is associated with a proinflammatory Th1 and Th17 cytokine profile. Cytokine. 2018; 104: 8-13.

20. Wong CK, Lam CW, Wu AK, Ip WK, Lee NL, Chan IH, et al. Plasma inflammatory cytokines and chemokines in severe acute respiratory syndrome. Clin Exp Immunol. 2004; 136(1): 95-103.

21. Yajing $F$, Cheng $Y$, Wu Y. Understanding SARS-CoV-2-mediated inflammatory responses: from mechanisms to potential therapeutic tools. Virol Sin. 2020.

22. Kikkert M. Innate immune evasion by human Respiratory RNA Viruses. J Innate Immun. 2020; 12(1): 4-20.

23. Channappanavar R, Perlman S. Pathogenic human coronavirus infections: causes and consequences of cytokine storm and immunopathology. Semin Immunopathol. 2017; 39(5): 529-39.

24. Shokri S, Mahmoudvand S, Taherkhani R, Farshadpour F. Modulation of the immune response by Middle East respiratory syndrome coronavirus. J Cell Physiol. 2019; 234(3): 2143-51.

25. Zhang L, Zhang F, Yu W, He T, Yu J, Yi CE, et. al. Antibody responses against SARS coronavirus are correlated with disease outcome of infected individuals. J Med Virol. 2006; 78: 1-8.

26. Wu Z, McGoogan JM. Characteristics of and important lessons from the coronavirus disease 2019 (COVID-19) outbreak in China: summary of a report of 72314 cases from the Chinese Center for Disease Control and Prevention. JAMA. 2020.

27. Zheng M, Gao Y, Wang G, Song G, Liu S, Sun D, et al. Functional exhaustion of antiviral lymphocytes in COVID-19 patients. Cell Mol Immunol. 2020.

28. Lippi G, Simundica AM, Plebania M. Potential preanalytical and analytical vulnerabilities in the laboratory diagnosis of coronavirus disease 2019 (COVID-19). Clin Chem Lab Med. 2020.

29. Deng Y, Lan J, Bao L, Huang B, Ye F, Chen Y, et al. Enhanced protection in mice induced by immunization with inactivated whole viruses compare to spike protein of middle east respiratory syndrome coronavirus. Emerg Microbes Infect. 2018; 7(1): 60.

30. Modjarrad K, Roberts CC, Mills KT, Castellano AR, Paolino K, Muthumani $\mathrm{K}$, et al. Safety and immunogenicity of an anti-Middle East respiratory syndrome coronavirus DNA vaccine: a phase 1, open-label, single-arm, dose-escalation trial. Lancet Infect Dis. 2019; 19(9): 1013-22.
31. Lin JT, Zhang JS, Su N, Xu JG, Wang N, Chen JT, et al. Safety and immunogenicity from a phase I trial of inactivated severe acute respiratory syndrome coronavirus vaccine. Antivir Ther. 2007; 12(7): 1107-13.

32. Lanese N, Writer S. First coronavirus vaccine trial in the US is recruiting volunteers. Live Science. 2020. Disponible en: https: // www.livescience.com/us-coronavirus-vaccine-trial-recruiting. html

33. Ying T, Du L, Ju TW, et al. Exceptionally potent neutralization of Middle East respiratory syndrome coronavirus by human monoclonal antibodies. J Virol. 2014; 88(14): 7796-7805.

34. Tseng C-TK, Huang C, Newman P, Wang N, Narayanan K, Watts DM, et al. Severe acute respiratory syndrome coronavirus infection of mice transgenic for the human Angiotensin-converting enzyme 2 virus receptor. J Virol. 2007; 81(3): 1162-73.

\section{Correspondencia:}

Joel de León Delgado

Dirección: Av. Alameda del Corregidor 1531, Urb. Los Sirius, La Molina. Lima, Perú.

Teléfono: 3652300 (anexo 147)

Correo electrónico: jdeleond@usmp.pe

\section{Recibido: 25 de marzo de 2020.}

Evaluado: 26 de marzo de 2020. Aprobado: 01 de abril de 2020 .

(c) La revista. Publicado por Universidad de San Martín de Porres, Perú. (c) BY Licencia de Creative Commons Artículo en acceso abierto bajo términos de Licencia Creative Commons Atribución 4.0 Internacional. (http://creativecommons.org/licenses/by/4.0/)

ORCID iDs

Joel De León Delgado

Arturo Pareja Cruz

Yanina Enriquez Valencia

Carlos Quiroz Carrillo

Edward Valencia Ayala
Chttps: / / orcid.org/0000-0002-3664-8023

- https: / / orcid.org/0000-0002-5988-5515

(ㄱ) https: / / orcid.org/0000-0002-8501-0915

(1) https: / / orcid.org/0000-0002-4821-9496

(ㄱ) https: / / orcid.org/0000-0002-5318-5526

Horiz Med (Lima) 2020; 20(2): e1209 